\title{
BMJ Open School reintegration following hospitalisation for children with medical complexity and chronic disease diagnoses: a scoping review protocol
}

\author{
Samantha Burns (D) , ${ }^{1}$ Katie Doering, ${ }^{1}$ Donna Koller, ${ }^{2}$ Catherine Stratton (D) ${ }^{3}$
}

To cite: Burns S, Doering K, Koller D, et al. School reintegration following hospitalisation for children with medical complexity and chronic disease diagnoses: a scoping review protocol. BMJ Open 2021;0:e052493. doi:10.1136/ bmjopen-2021-052493

- Prepublication history and additional supplemental material for this paper are available online. To view these files, please visit the journal online (http://dx.doi.org/10.1136/ bmjopen-2021-052493)

Received 17 April 2021 Accepted 30 September 2021

Check for updates

(c) Author(s) (or their employer(s)) 2021. Re-use permitted under CC BY-NC. No commercial re-use. See rights and permissions. Published by BMJ.

${ }^{1}$ Applied Psychology and Human Development, University of Toronto, Toronto, Ontario, Canada

${ }^{2}$ Early Childhood Studies, Ryerson University, Toronto, Ontario, Canada

${ }^{3}$ Chronic Disease Epidemiology, Yale University, New Haven, Connecticut, USA

Correspondence to

Ms. Samantha Burns;

samantha.burns@utoronto.ca

\section{ABSTRACT}

Introduction Schools play a significant role in children's social, emotional and intellectual well-being. For children with medical complexity (CMC) and chronic disease diagnoses (CDD), an absence from school due to prolonged hospitalisation places them at risk for greater social exclusion and poorer academic outcomes than their healthy counterparts. Processes that support the school reintegration of children with complex and chronic medical conditions currently lack consistency and identified evidence-based practices. This scoping review aims to integrate the relevant literature on current reintegration procedures as well as assess stakeholders' perceived challenges related to children with CMC and CDD's return to school following hospitalisation. Finally, information will be synthesised regarding parental and child involvement in reintegration strategies.

Methods and analysis The current scoping review follows the five-stage framework proposed by Arksey and 0'Malley (2005). The search syntax will be applied in Medline, Web of Science, Psyclnfo, Education Resource, ERIC, CINAHL and SocIndex. Peer-reviewed journal articles will be included without the restriction of publication year or language. However, only children and adolescents aged 4-18 with CMC and CDD, who have been out of school for 2 weeks or more and reintegrated into a non-hospital school setting will be included. Articles will be screened by two authors based on the outlined eligibility criteria. Data will be summarised qualitatively and where applicable, visualisation techniques such as tables, graphs and figures will be implemented to address approaches, strategies and outcomes related to reintegration to school following hospitalisation.

Ethics and dissemination The current study comprises available publications and does not collect primary data. For this reason, ethics approval is not necessary. The results of this scoping review will be prepared and submitted for publication in a peer-reviewed journal and presented at future conferences to key stakeholders focusing on educational accessibility and inclusion.

\section{BACKGROUND}

Children's development and well-being are largely influenced by their environment ${ }^{12}$ and globally, over one billion children spend the majority of their day attending either a
Strengths and limitations of this study

Novel comprehensive review approach reaching literature not included in previous reviews.

- The inclusion of articles will be published from English speaking journal articles.

- This review is limited by the information shared by the authors in terms of barriers and facilitators of school reintegration programmes.

primary or secondary school. ${ }^{3}$ Therefore, investigating the experiences of children in schools has important implications for research and policy. Research has shown that attending school leads to improved emotional regulation and the development of prosocial behaviours, likely attributable to the opportunities for social and intellectual stimulation. ${ }^{4}$ Time out of school, in contrast, has shown to have detrimental effects on children's mental health, well-being and educational outcomes. ${ }^{4}$ Furthermore, children who are absent from school due to illness are at an increased risk of experiencing negative outcomes. ${ }^{5}$

Numerous studies have addressed the importance of reintegration following hospitalisation of children. For example, Clemens and colleagues found that adolescents who required specific accommodations or modifications to schedules to perform optimally, faced stigma from peers and that these social challenges are best addressed proactively through the assignment of a school re-entry counsellor who can liaison the reintegration between hospital and school. ${ }^{6}$ Research has disproportionately focused on the importance of reintegration strategies for children with psychological disorders and a limited number of physical conditions, such as cancer, burn recovery and traumatic/ acquired brain injury. ${ }^{6-11}$ Additional research attention is required with children who have 
a chronic illness and/or medical complexity because they are more likely to require adequate planning and supportive processes for school reintegration. ${ }^{5}$

In 2018, there were 171786 hospitalisations among Canadians under the age of 18 , alone..$^{12}$ Of these children, there is a growing proportion who are characterised as children with medical complexity (CMC) who live with greater than one significant chronic health problem that, by definition, involves multiple organ systems resulting in multifaceted dysfunction, a significant need for healthcare services and, often, dependence on medical technologies. ${ }^{13-19}$ Collectively, research suggests that $\mathrm{CMC}$ medical conditions cause them to miss greater than 15 days of school within one academic year. ${ }^{5}$ Another classification of children at risk of pervasive hospitalisation is children with chronic disease(s) diagnoses (CDDs). A major definition for CDDs is outlined by Wijlaars et al as 'any health problem requiring clinical follow-up for $>12$ months in $50 \%$ or more of cases' ${ }^{20}$ Children with CDDs have almost a $35 \%$ increased risk of precarious social and academic development. ${ }^{21}$ The origin of these risks that children with physical health conditions experience are distinct from children with developmental disabilities and merit separate investigation and solutions ${ }^{22}$ as hospitalisation and time absent from school has unique implications for children's psychosocial well-being and developmental outcomes. ${ }^{23}$

For example, CMC and CDD use medical technologies for their survival and health management. An important consideration for this population is the degree of self-management CMCs possess and to what extent educators and school administrators can be expected to play a role in the management of CMCs' ongoing health management needs. One study reviewed literature on CMC by applying The Pediatric Self-Management Model, ${ }^{184}$ which can be used to ascertain patients' ability to manage their own care needs through greater understanding of their condition(s). Important predictors for improved adjustment to new medical technologies were identified, such as whether families of CMC viewed the technology as a 'puzzle' to solve versus a burden. ${ }^{18}$ Given that most educators of CMC will not be trained to operate various medical technologies, this research highlights the importance of having school reintegration plans that are tailored to each student's needs and circumstances. These plans should consider the level of ongoing care management and the degree to which the child can handle their care independently.

A preliminary scoping review helped identify relevant variables in the assessment of hospital-to-home transition for CMC. ${ }^{19}$ The investigators elected to assess stakeholder perceptions on the usefulness of transition plans since while there are numerous benefits of transition of care plans, it is possible that stakeholders find them less effective in the CMC population given the regularity and unpredictability of rehospitalisation. An important finding from this scoping review was that all included studies concluded that transition of care plans are useful in optimising communications between caregivers and healthcare providers. ${ }^{19}$ Next steps could include determining if the purported improved communication extends to involved educators in this study.

The impetus for this scoping review is based on a lack of national school reintegration programmes or standards for CMC. There are potentially many reasons for this discrepancy. One reason may be more practical, such as the lack of provincial funding for such an initiative. Other factors though shed light on the complexity of such an undertaking. The range of medical conditions and circumstances for which school-aged children might require hospitalisation cannot be universally addressed in a singular programme or protocol. A major priority for children who have been hospitalised for an extensive period of time is a successful school reintegration. The physical and psychological effects of hospitalisation will vary from child to child creating individual challenges to reintegration. For example, a child who was hospitalised once carries the burdensome trauma attached to that event as well as the challenge of learning all of the curricula they missed during hospitalisation. Nevertheless, if such a student is otherwise healthy, it is unlikely that the student will face further hospitalisations. In this case, school reintegration will likely be a top priority for the student's educational and health teams and be a more straightforward process. In children with chronic and recurring illnesses though, there is added complexity in predicting future hospitalisations and reintegrations as well as the additive trauma and growing disruptions to a child's education from serial hospitalisations. Previous research has demonstrated that CMC are frequently hospitalised. In fact, some research has suggested that the 30-day hospital readmission rate for CMC was nearly $24 \%{ }^{19}$ This further supports the aims of this scoping review which will inform school reintegration protocols for this unique population.

While there is existing literature on the reintegration of students for certain causes for hospitalisation, such as burns and cancer, ${ }^{9} 10$ the current literature does not encompass the range of $\mathrm{CMC}$ and $\mathrm{CDD}$ and their unique experience. These complexities also extend to academic support and gauging who is responsible for planning and executing the transition. An important tenet of school reintegration identified in the psychological literature is the executive coordination among all relevant medical and educational professionals providing support to children. ${ }^{623}$

Some children with complex educational needs have academic supports in place involving teaching staff who have the training to work with such students. ${ }^{25}$ By contrast, a child with a physical illness, but no learning or intellectual disabilities, may return to their standard learning environment without the required resources to make their return seamless. Another explanation for the lack of a comprehensive school reintegration programme is that medical advances have outpaced the stakeholders involved in the design of such a programme. Currently, over $80 \%$ of children with cancer will survive and the survival of certain individual cancers is far higher 
than that rate. ${ }^{10}$ In the past, it may have been feasible for healthcare providers and educators to ignore the relevance of school integration when many critically ill children did not survive ${ }^{10}$ For this reason, it is timely to advocate for best practices that support children's lives post-hospitalisation.

While there have been efforts to design school reintegration programmes at a local or individual level, their results and success have seldom been assessed cumulatively; a necessary step to delineate the evidence to inform a broader reintegration programme. Canter et al conducted a systematic review in 2012 to assess school re-entry in children with chronic illness. However, the final analysis only featured content on four medical conditions and $75 \%$ of the papers were solely focused on cancer. ${ }^{10}$ Other research has shown that school reintegration has been oversaturated with the assessment of patients with cancer, burns and other major causes of illness. ${ }^{9-1123}$ This has created a notable gap in the literature on school reintegration for patients with other, less-studied conditions, such as CMC. To address this knowledge gap, our investigation will examine this by expanding the inclusion criteria with a focus on CMC.

\section{METHODS AND ANALYSIS Protocol design}

The current protocol was developed using the methodological framework proposed by Arksey and O'Malley $(2005)^{26}$ and further examined by Joanna Briggs Institute. ${ }^{27}$ In addition, this protocol follows the Preferred Reporting Items for Systematic Reviews and MetaAnalyses extension for Scoping Review (PRISMA ScR; online supplemental appendix 1). The study search will be conducted in November 2021 and the project is expected to be completed in June 2022.

\section{Stage 1: identify the research question}

An environmental scan of the literature was conducted to inform our research questions. For the purpose of this review, we will focus on children (4-18 years) following discharge who reintegrate into an out-of-hospital school setting.

Based on the initial exploration of the literature, the following research questions were developed:

1. What are current practices of reintegration into the school setting of hospitalised children with CMC and CDD? When are these practices typically applied (ie, before and/or during hospitalisation)? Which practices have been effective in CMC and CDD if any?

2. How is school reintegration planned and what steps are taken to optimise the execution of school reintegration plans? How do stakeholders manage follow-up of reintegration plans?

3. What are the reported barriers to reintegration into the school setting of hospitalised children with CMC and CDD?
4. Who is responsible for the reintegration planning and implementation process? Who are the key stakeholders?

5. Are parents involved in reintegration planning and/or implementation? What role do parents play?

6. Are children involved in reintegration planning and/ or implementation? What role do children assume? Based on a child's right to participate in decisions, how are they involved in the planning, execution and follow-up stages?

\section{Stage 2: search for relevant studies}

Following Arksey and O'Malley's ${ }^{26}$ framework, the second stage of the scoping review aimed to identify and develop inclusion criteria to be used when selecting studies for the review. These criteria helped inform the search syntax.

The scoping review will include published studies from the following databases: Medline, PsycInfo, Web of Science, Education Resource, ERIC, CINAHL and SocIndex. Reference lists of relevant studies will be checked to ensure that all applicable articles will be included.

Based on the initial exploratory research, the following eligibility criteria were implemented:

- Type of publication: journal articles.

- Publication year: any.

- Language: all.

- Study population: children and adolescents, aged 4-18 years.

- Types of articles: primary studies, systematic reviews, meta-analyses, scoping reviews, evidence maps, rapid reviews, literature reviews, evidence syntheses, reviews of reviews, narrative reviews and critical reviews.

- Setting: out-of-hospital school settings.

- Time away from hospital: 2 weeks or more. ${ }^{5}$

An academic librarian trained in review strategies was consulted regarding the most appropriate subject heading terms and how to modify them across databases. The search syntax for each database was finalised and included terms concerning the most frequently studied medical conditions such as 'Juvenile', 'Burns', 'Neoplasms', 'Arthritis', 'Congenital' and 'Hereditary'. Broader terms were applied to capture other conditions such as 'Neonatal disease', 'Abnormalities', 'Exceptional', 'Disease', 'Disorder', 'Serious illness', 'Complex medical', 'Chronic disease', 'Multiple chronic conditions', 'Medical complexity', 'Surgery', 'Traumatic', 'Paediatric' and 'Paediatric' were included. To search for the study population, the terms 'High School', 'Middle School', 'Preschool', 'Kindergarten', 'Elementary School', 'Nursing School', 'Day care', 'School' and 'Education' were included. To search for research involving key stakeholders in school reintegration for CMC, the terms 'Student', 'Patient', 'Doctor', 'Physician', 'Nurse', 'Occupational Therapist', 'Speech Therapist', 'Speech Language Pathologist', 'Child and Youth Workers', 'Teacher', 'Principal', 'Early Childhood Educator', 'Teaching Assistant', 'Social Worker', 'Psychologist', 'Multidisciplinary', 'Interdisciplinary', 'Cross-professional', 'Collaboration', 'School Liaison', 
'Hospital Educator', 'Child Life', 'School Nurse', 'Parent', 'Guardian' and 'Caretaker' were included. Finally, reintegration and transition terms such as 'Re-entry', 'Reintegration', 'Transition', 'Return to' and 'Education' were included. Articles will then be retrieved from each database and imported into Covidence, the online systematic review platform. For an example of the search syntax for reintegration and the stakeholders applied in the database Medline, please see online supplemental appendices 2 and 3 , respectively.

\section{Stage 3: selection of relevant studies}

The third stage of Arksey and O'Malley's ${ }^{26}$ framework aims to identify the selection of relevant studies. Following the consolidation of articles generated from the searches across databases, duplicates will be removed. Two members of the team (SB, CS) will independently screen the titles and abstracts of all articles to determine which articles meet the eligibility criteria devised in the second stage. Following the abstract screening, full texts will be retrieved for full-text review. Disagreements

\section{Table 1 Variables to chart}

\begin{tabular}{ll}
\hline Main category $\quad$ Subcategory & Description \\
\hline Authors & \\
Title & \\
Year &
\end{tabular}

Journal

\section{Country}

$\begin{array}{ll}\text { Age of children } & \text { Mean age } \\ & \text { The age range }\end{array}$

Number of children

Grade

School type

\section{Classroom type}

Diagnosis

Length of diagnosis

Reason for absence

Limitations/accommodation

Length of absence

Length between hospital and school

Number of

hospitalisations

$\begin{array}{ll}\text { Integration procedure } & \begin{array}{l}\text { Professional facilitating } \\ \text { integration }\end{array} \\ & \text { Length of integration } \\ & \text { Process of integration }\end{array}$

Child involvement

Parent/caregiver involvement

Reintegration plan

\begin{tabular}{|c|c|}
\hline \multirow[t]{4}{*}{ Outcome of integration } & $\begin{array}{l}\text { Type of outcome } \\
\text { investigated }\end{array}$ \\
\hline & Description of outcome \\
\hline & Barriers to integration \\
\hline & $\begin{array}{l}\text { Key facilitators of } \\
\text { integration }\end{array}$ \\
\hline
\end{tabular}

Country data collected from in the study

The arithmetic average of all children included

Difference between oldest and youngest child

Total number of children included in the study

School grade

Type of school child is attending (ie, out-of-hospital school, public school, private school)

Type of classroom children were integrated into

The medical diagnosis of a child

How long the child has had the diagnosis

The reason that child had to be away or postponed from attending school

Functional limitations and/or required accommodations on reintegration into school

Length of time child was removed from school

Length of time between hospitalisation and reintegration (ie, time spent at home and details pertaining to homebound instruction if pertinent to the student's reintegration plan)

Number of hospitalisations a student has experienced (or is anticipated to experience) over the past academic year causing absence from school for 2 weeks or longer each time

The professional members of the team responsible for the integration of the child (ie, child-life, paediatrician, social worker)

Length of time integration into school took place

Procedure or process of integration (ie, planned before or implemented during hospitalisation)

Child's role in their integration into school and steps taken to ensure a patient-centred approach

Parent/caregiver's role in their child's integration into school

Details on accommodations implemented to support school reintegration including if the plan address all accommodation needs and if the plan involved the use of interactive technologies (ie, robots, tele-education and so on)

Determinant(s) of successful integration (ie, child well-being, academic success)

The information provided on the outcome of integration as defined in the study Reported barriers to integration

Key aspects that enhanced the success of integration into the school setting including incorporating student perspectives into the reintegration plan 
about study eligibility will be discussed between the two reviewers until a consensus is reached. If a consensus is unable to be reached, a third party will be consulted (DK, $\mathrm{KD})$. Study selection is reported using a PRISMA ScR flow chart taken from Tricco and colleagues (2018) and will be updated once each stage is complete. ${ }^{28}$

\section{Stage 4: Charting the data}

Based on the preliminary investigation of the data, 16 categories have been identified that will be used for the literature screening when determining the inclusion of the articles (table 1). For each article, descriptive information (ie, title, author, journal and year of publication) and type of publication will be extracted independently by two authors (SB, CS). Furthermore, participant characteristics will be collected. Information regarding the process and barriers to reintegration will be tabled. The framework will be pilot tested by two authors on a sample of the study to ensure coding is extracted consistently. If there are discrepancies, then the framework will be revised accordingly. Questions related to the extraction procedure will be discussed and disagreements will be resolved through team consultations.

Following training and agreement by the team members, independent reviews of the articles and extraction will occur. To ensure inter-rater reliability, a sample of $20 \%$ of the articles will be double reviewed and discussion will occur between the two reviewers. Discrepancies in extracted data will be discussed and if consensus cannot be reached a third party will be included.

\section{Stage 5: Summarising the results}

Following the extraction of data, statistical and thematic analysis of results will be conducted to summarise the current literature pertaining to school reintegration among CMC. In particular, the common challenges within school reintegration and barriers to successful reintegration will be presented. Similarly, constituents of school reintegration following hospitalisation and evidence-based protocols will be discussed and assessed per feasibility for implementation. Important stratification of results (by age/grade, diagnosis and school characteristics) will be performed to ensure that the suggestions for reintegration guidelines are accurate and effective. Furthermore, the identification of effective tenets of reintegration will also expose domains that remain understudied and merit continued investigation. The results will be presented through a combination of descriptive analysis, tables, charts, figures and other visual tools as needed.

\section{Ethics and dissemination}

The current study comprises available publications and does not collect primary data. Therefore, this study does not require formal ethics approval from the Research Ethics Board. The results of this scoping review will be prepared and submitted for publication in a peerreviewed journal for readership by key stakeholders (eg, patients, parents, teachers, physicians, social workers, child and youth workers, nurses, child life specialists and speech language pathologists) and presented at future conferences focusing on educational accessibility and inclusion of CMC. Furthermore, this scoping review will form the basis of identifying evidence-based practice for school reintegration and policy and future research initiatives.

\section{Patient and public involvement}

No patients were involved in the preparation of this scoping review protocol but will be engaged during future stages of this research. Experts in the field were involved in the development of research questions and outcomes, further to consultation with their interdisciplinary colleagues who work with CMC and CDD and their families. To ensure patient and family perspectives are highlighted, we have included child and family involvement in reintegration plan formation. The authors plan to disseminate the results to patients and families by sharing the results with local paediatric hospitals, school authorities, public and private schools that serve CMC and CDD, school bridging programme practitioners and preservice educators. This scoping review is the first step to form a best practice guideline for school reintegration following hospitalisation.

Twitter Samantha Burns @Samanth21984892 and Catherine Stratton @cathsmstratton

Contributors SB contributed to the development of the research questions, drafting and editing this protocol and substantially contributed to the development of the methods. KD contributed to the conceptual framework, methods and edited the protocol. DK contributed to the conceptual framework, methods and edited the protocol. CS contributed to the development of the research questions and extensively drafted and edited the protocol. All authors have approved the final manuscript.

Funding The authors have not declared a specific grant for this research from any funding agency in the public, commercial or not-for-profit sectors.

Competing interests None declared.

Patient consent for publication Not applicable.

Provenance and peer review Not commissioned; externally peer reviewed.

Data availability statement Data sharing not applicable as no datasets generated and/or analysed for this study.

Supplemental material This content has been supplied by the author(s). It has not been vetted by BMJ Publishing Group Limited (BMJ) and may not have been peer-reviewed. Any opinions or recommendations discussed are solely those of the author(s) and are not endorsed by BMJ. BMJ disclaims all liability and responsibility arising from any reliance placed on the content. Where the content includes any translated material, BMJ does not warrant the accuracy and reliability of the translations (including but not limited to local regulations, clinical guidelines, terminology, drug names and drug dosages), and is not responsible for any error and/or omissions arising from translation and adaptation or otherwise.

Open access This is an open access article distributed in accordance with the Creative Commons Attribution Non Commercial (CC BY-NC 4.0) license, which permits others to distribute, remix, adapt, build upon this work non-commercially, and license their derivative works on different terms, provided the original work is properly cited, appropriate credit is given, any changes made indicated, and the use is non-commercial. See: http://creativecommons.org/licenses/by-nc/4.0/.

ORCID iDs

Samantha Burns http://orcid.org/0000-0001-5251-0369

Catherine Stratton http://orcid.org/0000-0002-1841-4565 


\section{REFERENCES}

1 Bronfenbrenner U, Morris PA. The ecology of developmental processes. In: Handbook of child psychology: theoretical models of human development. New York, NY: John Wiley \& Sons Inc, 1998: 993-1028.

2 Yoshikaw H, Weiland C, Brooks-Gunn J. When does preschool matter? Future Child 2016;26:21-35.

3 UNICEF. Education, 2020. Available: www.unicef.org/education/ accessed [Accessed 13 Feb 2021].

4 Morgan K, Melendez-Torres G, Bond A, et al. Socio-economic inequalities in adolescent summer holiday experiences, and mental wellbeing on return to school: analysis of the school health research network/health behaviour in school-aged children survey in Wales. Int J Environ Res Public Health 2019;16:1107.

5 Allison MA, Attisha E, COUNCIL ON SCHOOL HEALTH. The link between school attendance and good health. Pediatrics 2019;143:e20183648.

6 Clemens EV, Welfare LE, Williams AM. Tough transitions: mental health care professionals' perception of the psychiatric hospital to school transition. Resid Treat Child Youth 2010;27:243-63.

7 Tougas A-M, Rassy J, Frenette-Bergeron Émilie, et al. "Lost in transition": a systematic mixed studies review of problems and needs associated with school reintegration after psychiatric hospitalization. School Ment Health 2019;11:629-49.

8 Clemens EV, Welfare LE, Williams AM. Elements of successful schoo reentry after psychiatric hospitalization. Preventing School Failure: Alternative Education for Children and Youth 2011;55:202-13.

9 Arshad SN, Gaskell SL, Baker C, et al. Measuring the impact of a burns school reintegration programme on the time taken to return to school: a multi-disciplinary team intervention for children returning to school after a significant burn injury. Burns 2015;41:727-34.

10 Canter KS, Roberts MC. A systematic and quantitative review of interventions to facilitate school reentry for children with chronic health conditions. J Pediatr Psychol 2012;37:1065-75.

11 Lindsay S, Hartman LR, Reed N, et al. A systematic review of hospital-to-school reintegration interventions for children and youth with acquired brain injury. PLoS One 2015;10::e0124679.

12 Canadian Institute for Health Information. Almost 12,000 Canadian children and youth readmitted to hospital last year. CIHI, 2019. Available: www.cihi.ca/en/almost-12000-canadian-children-andyouth-readmitted-to-hospital-last-year/accessed [Accessed 13 Feb 2021]

13 Kuo DZ, Houtrow AJ, COUNCIL ON CHILDREN WITH DISABILITIES. Recognition and management of medical complexity. Pediatrics 2016;138:e20163021.
14 Cohen E, Patel $\mathrm{H}$. Responding to the rising number of children living with complex chronic conditions. Can Med Assoc J 2014;186:1199-200.

15 Cohen E, Berry JG, Sanders L, et al. Status complexicus? the emergence of pediatric complex care. Pediatrics 2018;141:S202-11.

16 Cohen E, Kuo DZ, Agrawal R, et al. Children with medical complexity: an emerging population for clinical and research initiatives. Pediatrics 2011;127:529-38.

17 Kuo DZet al. A national profile of caregiver challenges among more medically complex children with special health care needs. Arch Pediatr Adolesc Med 2011;165:1020-6.

18 Trowbridge K, Mische-Lawson L. Families with children with medical complexity and self-management of care: a systematic review of the literature. Soc Work Health Care 2014;53:640-58.

19 Breneol S, Belliveau J, Cassidy C, et al. Strategies to support transitions from hospital to home for children with medical complexity: a scoping review. Int J Nurs Stud 2017;72:91-104.

20 Wijlaars LPMM, Gilbert R, Hardelid P. Chronic conditions in children and young people: learning from administrative data. Arch Dis Child 2016;101:881-5.

21 Bell MF, Bayliss DM, Glauert R, et al. Chronic illness and developmental vulnerability at school entry. Pediatrics 2016;137:e20152475.

22 Irwin MK, Elam M. Are we leaving children with chronic illness behind? Physical Disabilities: Education and Related Service 2011;30:67-99 https://eric.ed.gov/?id=EJ955447

23 Lum A, Wakefield CE, Donnan B, et al. Understanding the school experiences of children and adolescents with serious chronic illness: a systematic meta-review. Child: Care Health and Development 2017;43:645-62.

24 Modi AC, Pai AL, Hommel KA, et al. Pediatric self-management: a framework for research, practice, and policy. Pediatrics 2012;129:e473-85.

25 Breaz AM, Goian C. Recovery of children with intellectual disabilities in the mass school. Educația-plus 2019;23:87-96 https://www.uav.ro/ jour/index.php/jpe/article/view/1265/1352

26 Arksey H, O'Malley L. Scoping studies: towards a methodological framework. Int J Soc Res Methodol 2005;8:19-32.

27 Peters MDJ, Godfrey CM, Khalil H, et al. Guidance for conducting systematic scoping reviews. Int J Evid Based Healthc 2015;13:141-6.

28 Tricco AC, Lillie E, Zarin W, et al. PRISMA extension for scoping reviews (PRISMA-scr): checklist and explanation. Ann Intern Med 2018;169:467-73. 